\title{
A Concise Asymmetric Route to Nuphar Alkaloids. A Formal Synthesis of (-)-Deoxynupharidine
}

\author{
Wesley J. Moran, ${ }^{\dagger}$ Katharine M. Goodenough, ${ }^{\dagger}$ Piotr Raubo ${ }^{\Uparrow}$ and Joseph P. A. Harrity ${ }^{\dagger}$ \\ ${ }^{\dagger}$ Department of Chemistry, University of Sheffield, S3 7HF, United Kingdom \\ "The Neuroscience Research Centre, Merck, Sharp and Dohme, Terlings Park, Harlow, \\ Essex, CM20 2QR
}

\section{Supporting Information}

\section{Table of Contents}

Experimental Section

Experimental Data for 6

Experimental Data for 10

Experimental Data for 11

${ }^{1} \mathrm{H}$ NMR Spectrum of 16 


\section{Experimental Section}

\section{General}

Infrared (IR) spectra were recorded on a Perkin Elmer Paragon 100 FTIR spectrophotometer, $v_{\max }$ in $\mathrm{cm}^{-1}$. Bands are characterised as broad (br), strong (s), medium (m) and weak (w). ${ }^{1} \mathrm{H}$ NMR spectra were recorded on a Bruker AC-250 (250 MHz). Chemical shifts are reported in ppm from tetramethylsilane with the solvent resonance as the internal standard $\left(\mathrm{CHCl}_{3}: \delta 7.27 \mathrm{ppm}\right)$. Data are reported as follows: chemical shift, integration, multiplicity ( $\mathrm{s}=$ singlet, $\mathrm{d}=$ =doublet, $\mathrm{t}=$ triplet, $\mathrm{q}=$ quartet, $\mathrm{br}=$ broad, $\mathrm{m}=$ multiplet) and coupling constants $(\mathrm{Hz}) .{ }^{13} \mathrm{C}$ NMR spectra were recorded on a Bruker AC-250 (62.9 MHz) with complete proton decoupling. Chemical shifts are reported in ppm from tetramethylsilane with the solvent resonance as the internal standard $\left(\mathrm{CDCl}_{3}: \delta 77.0 \mathrm{ppm}\right)$. Elemental microanalysis was performed using a Perkin Elmer $2400 \mathrm{CHN}$ elemental analyser, for anions (S, Cl) classical wet analysis was performed. Low resolution mass spectra $(\mathrm{m} / \mathrm{z})$ were recorded on either a Kratos MS 25 or MS 80 spectrometer supported by a DS 55 data system, operating in E.I., C.I. or positive FAB mode, with only molecular ions $\left(\mathrm{M}^{+}\right)$being reported. High resolution mass spectra were obtained using a Kratos MS 80 spectrometer supported by a DS 90 data system. Optical rotations were recorded on a Perkin Elmer 241 automatic polarimeter at $589 \mathrm{~nm}$ (Na D-line) with a path length of $1 \mathrm{dm}$. Concentrations (c.) are quoted in $\mathrm{g} / 100 \mathrm{ml}$. Melting points (m.p.) were recorded on a Gallenkamp melting point apparatus and are uncorrected.

All reactions were conducted in oven and flame-dried glassware under an inert atmosphere of dry nitrogen. All reagents were used as received from commercial suppliers unless otherwise stated. Dichloromethane, toluene, $N, N, N^{\prime}, N^{\prime}$-tetramethylethylenediamine and triethylamine were distilled from calcium hydride. Tetrahydrofuran and diethyl ether were distilled from sodium metal/benzophenone ketal. Dimethylsulfoxide was distilled from calcium hydride under reduced pressure. 2-Methyl-2-propen-1-ol was distilled from potassium carbonate. Methanol was distilled from magnesium. Petroleum ether was distilled over molecular sieves. Flash chromatography was performed on silica gel (Merck Kieselgel 60 $\mathrm{F}_{254}$ 230-400 mesh) according to the method of W.C. Still. Thin layer chromatography (TLC) was performed on aluminium backed plates pre-coated with silica $(0.2 \mathrm{~mm}$, Merck DCalufolien Kieselgel $60 \mathrm{~F}_{254}$ ) which were developed using standard visualising agents.

(2R)- $N$-(p-Toluenesulfonyl)-aspartic acid dimethyl ester 7 was prepared in two steps according to literature methods and showed satisfactory analytical and spectroscopic data. ${ }^{1}$ 
Synthesis of $(2 R, 3 S)-N$-(p-toluenesulfonyl)-aspartic acid dimethyl ester, 8.

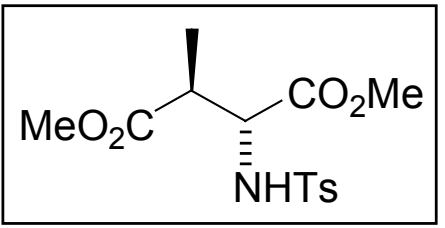

A solution of diester $7(1.25 \mathrm{~g}, 4.0 \mathrm{mmol})$ in THF (12 ml) was added via cannula to a stirred solution of $1.06 \mathrm{M}$ lithium hexamethyldisilazane in THF (LiHMDS, $8.7 \mathrm{ml}, 8.7 \mathrm{mmol}$ ) precooled to $-78{ }^{\circ} \mathrm{C}$ under a nitrogen atmosphere. After $2 \mathrm{~h}$, iodomethane $(0.3 \mathrm{ml}, 4.8 \mathrm{mmol})$ was added slowly to the reaction mixture, and stirring was continued for a further $15 \mathrm{~h}$. The mixture was quenched by the addition of $3 \mathrm{~N} \mathrm{HCl}$ to $\mathrm{pH} \sim 3$, and the aqueous layer was saturated with sodium chloride and then extracted with EtOAc $(10 \mathrm{ml} \times 2)$. The combined organic layer was dried over $\mathrm{MgSO}_{4}$ and evaporated under reduced pressure to give an oily residue. Purification by column chromatography (silica gel, 2:1 petroleum ether/EtOAc) gave a white solid (1.17 g, 89\%):

Mp: $73.5-75.5^{\circ} \mathrm{C}$.

$[\alpha]_{D}=-24\left(c=1.0, \mathrm{CH}_{2} \mathrm{Cl}_{2}\right)$.

IR $\left(\mathrm{CH}_{2} \mathrm{Cl}_{2}\right): 3357$ (br), 3062 (w), $2956(\mathrm{~m}), 1742(\mathrm{~s}), 1344(\mathrm{~m}), 1166(\mathrm{~s}) \mathrm{cm}^{-1}$.

${ }^{1} \mathrm{H} \mathrm{NMR}\left(\mathrm{CDCl}_{3}\right): \delta 1.23(3 \mathrm{H}, \mathrm{d}, J=7.5 \mathrm{~Hz}), 2.38(3 \mathrm{H}, \mathrm{s}), 3.11(1 \mathrm{H}, \mathrm{dq}, J=4.0,7.5 \mathrm{~Hz}), 3.45$ $(3 \mathrm{H}, \mathrm{s}), 3.62(3 \mathrm{H}, \mathrm{s}), 4.05(1 \mathrm{H}, \mathrm{dd}, J=4.0, J=9.5 \mathrm{~Hz}), 5.60(1 \mathrm{H}, \mathrm{d}, J=9.5 \mathrm{~Hz}), 7.26(2 \mathrm{H}, \mathrm{d}$, $J=8.0 \mathrm{~Hz}), 7.70(2 \mathrm{H}, \mathrm{d}, J=8.0 \mathrm{~Hz})$.

${ }^{13} \mathrm{C} \operatorname{NMR}\left(\mathrm{CDCl}_{3}\right): \delta 13.6,21.5,42.3,52.2,52.6,57.8,127.3,129.5,137.0,143.6,170.4$, 173.5 .

Anal. Calcd for $\mathrm{C}_{14} \mathrm{H}_{19} \mathrm{NSO}_{6}$ : C, 51.05; $\mathrm{H}, 5.81 ; \mathrm{N}, 4.25 ; \mathrm{S}, 9.74$. Found: $\mathrm{C}, 50.92 ; \mathrm{H}, 5.76 ; \mathrm{N}$, $4.20 ;$ S, 9.82.

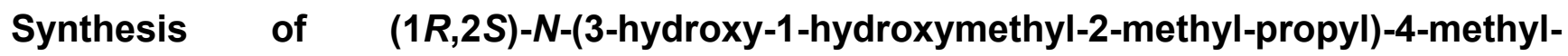
benzenesulfonamide, 9 .

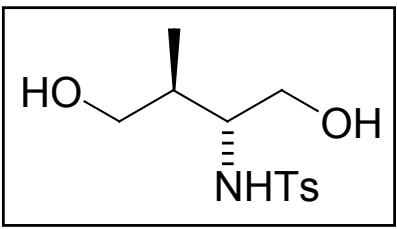

A solution of the diester $8(710 \mathrm{mg}, 2.2 \mathrm{mmol})$ in THF $(10 \mathrm{ml})$ was added dropwise via cannula to a solution of lithium aluminium hydride (164 mg, $4.3 \mathrm{mmol})$ in THF $(15 \mathrm{ml})$ at $0{ }^{\circ} \mathrm{C}$ under a nitrogen atmosphere, and stirred for $16 \mathrm{~h}$ at $\mathrm{rt}$. Aqueous potassium carbonate solution was added to quench the reaction and the resulting mixture extracted with ethyl acetate. The organic layer was dried over $\mathrm{MgSO}_{4}$ and evaporated under reduced pressure to give a white solid ( $575 \mathrm{mg}, 98 \%)$ :

Mp: $76.0-78.0^{\circ} \mathrm{C}$.

$[\alpha]_{D}=+7\left(c=1.0,\left(\mathrm{CH}_{3}\right)_{2} \mathrm{CO}\right)$. 
IR $\left(\mathrm{CH}_{2} \mathrm{Cl}_{2}\right)$ : 3359 (br), $2968(\mathrm{~m}), 2935$ (m), $1599(\mathrm{~m}), 1413$ (s), 1332 (s), 1161 (s), 1093 (s) $\mathrm{cm}^{-1}$.

${ }^{1} \mathrm{H}$ NMR $\left(\mathrm{CDCl}_{3}\right): \delta 0.86(3 \mathrm{H}, \mathrm{d}, J=7.0 \mathrm{~Hz}), 1.76-1.93(1 \mathrm{H}, \mathrm{m}), 2.42(3 \mathrm{H}, \mathrm{s}), 3.18-3.25(1 \mathrm{H}$, m), $3.31(1 \mathrm{H}, \mathrm{dd}, J=4.0,11.5 \mathrm{~Hz}), 3.45(1 \mathrm{H}, \mathrm{dd}, J=6.5,11.0 \mathrm{~Hz}), 3.56(1 \mathrm{H}, \mathrm{dd}, J=3.0$, $11.5 \mathrm{~Hz}), 3.75(1 \mathrm{H}, \mathrm{dd}, J=3.0,11.0 \mathrm{~Hz}), 7.30(2 \mathrm{H}, \mathrm{d}, J=8.0 \mathrm{~Hz}), 7.77(2 \mathrm{H}, \mathrm{d}, J=8.0 \mathrm{~Hz})$.

${ }^{13} \mathrm{C} \mathrm{NMR}\left(\mathrm{CDCl}_{3}\right): \delta 14.8,21.5,37.3,58.0,63.0,64.0,127.1,129.8,137.5,143.6$.

HRMS Calcd for $\mathrm{C}_{12} \mathrm{H}_{20} \mathrm{NSO}_{4}: 274.1113$. Found: 274.1115.

\section{Synthesis of 2(S)-[N-(p-toluenesulfonyl)-aziridin-2(S)-yl]-propan-1-ol, 6.}

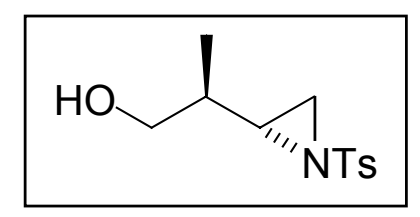

Tributylphosphine $(0.56 \mathrm{~g}, 2.7 \mathrm{mmol}, 1.5 \mathrm{eq}$.) was added to a solution of diol 9 (0.5 g, 1.8 mmol, 1 eq.) in toluene $(12 \mathrm{ml})$ at $\mathrm{rt}$ under a nitrogen atmosphere. The temperature was lowered to $0{ }^{\circ} \mathrm{C}$ and ADDP (0.69 g, $2.7 \mathrm{mmol}, 1.5 \mathrm{eq}$.) added portionwise. The reaction mixture was stirred for $20 \mathrm{~h}$ at $\mathrm{rt}$, then the solvent was removed in vacuo to give an oil. Careful chromatography provided a small sample of analytically pure material.

$[\alpha]_{\mathrm{D}}=-30\left(c=1.68, \mathrm{CH}_{2} \mathrm{Cl}_{2}\right)$.

IR ( $\left.\mathrm{CH}_{2} \mathrm{Cl}_{2}\right): 3417$ (br), 2964 (m), 2935 (m), 1736 (s), 1495 (s), 1220 (s), 1161 (s), 1061 (s) $\mathrm{cm}^{-1}$.

${ }^{1} \mathrm{H}$ NMR $\left(\mathrm{CDCl}_{3}\right): \delta 1.00(3 \mathrm{H}, \mathrm{d}, J=7.0 \mathrm{~Hz}), 1.41-1.55(1 \mathrm{H}, \mathrm{m}), 2.14(1 \mathrm{H}, \mathrm{d}, J=4.5 \mathrm{~Hz}), 2.46$ $(3 \mathrm{H}, \mathrm{s}), 2.61(1 \mathrm{H}, \mathrm{d}, J=7.0 \mathrm{~Hz}), 2.65-2.74(1 \mathrm{H}, \mathrm{m}), 3.50-3.58(2 \mathrm{H}, \mathrm{m}), 7.34(2 \mathrm{H}, \mathrm{d}, J=8.0$ $\mathrm{Hz}), 7.84(2 \mathrm{H}, \mathrm{d}, J=8.0 \mathrm{~Hz})$.

${ }^{13} \mathrm{C} \mathrm{NMR}\left(\mathrm{CDCl}_{3}\right): \delta 13.8,21.6,32.9,37.6,42.1,65.9,128.0,129.7,134.5,144.8$.

HRMS Calcd for $\mathrm{C}_{12} \mathrm{H}_{18} \mathrm{NSO}_{3}: 256.1007$. Found: 256.0995.

\section{Synthesis of 2(S)-[2-(tert-butyl-dimethyl-silanyloxy)-1(S)-methyl-ethyl]- $N$-(p-} toluenesulfonyl)-aziridine, 10.

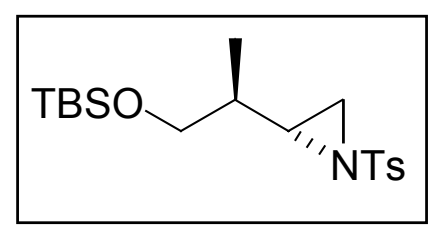

$t$-Butyldimethylsilyl chloride $(0.33 \mathrm{~g}, 2.2 \mathrm{mmol})$ and imidazole $(0.16 \mathrm{mg}, 2.3 \mathrm{mmol})$ were added to a solution of impure aziridine $6(0.46 \mathrm{~g})$ in dry THF $(10 \mathrm{ml})$ at $0{ }^{\circ} \mathrm{C}$ and stirred for 5 $\mathrm{h}$. The solvent was removed in vacuo and the residue purified by flash chromatography 
(silica gel, 8:1 petroleum ether/EtOAc) to give protected aziridine ( $0.61 \mathrm{~g}, 90 \%$ over 2 steps) as a white solid, which was recrystallised from petroleum ether:

Mp: $81.0-82.0^{\circ} \mathrm{C}$.

$[\alpha]_{\mathrm{D}}=-4\left(c=1.0, \mathrm{CH}_{2} \mathrm{Cl}_{2}\right)$

IR $\left(\mathrm{CH}_{2} \mathrm{Cl}_{2}\right): 2957(\mathrm{~m}), 2950(\mathrm{~m}), 2858(\mathrm{~m}), 1472(\mathrm{~m}), 1323(\mathrm{~m}), 1162(\mathrm{~s}) \mathrm{cm}^{-1}$.

${ }^{1} \mathrm{H}$ NMR $\left(\mathrm{CDCl}_{3}\right): \delta-0.05(6 \mathrm{H}, \mathrm{s}), 0.84(9 \mathrm{H}, \mathrm{s}), 0.89(3 \mathrm{H}, \mathrm{d}, J=6.5 \mathrm{~Hz}), 1.32-1.42(1 \mathrm{H}, \mathrm{m})$, $2.15(1 \mathrm{H}, \mathrm{d}, J=3.0 \mathrm{~Hz}), 2.43(3 \mathrm{H}, \mathrm{s}), 2.55-2.70(2 \mathrm{H}, \mathrm{m}), 3.21(1 \mathrm{H}, \mathrm{dd}, J=6.0 \mathrm{~Hz}, 9.5 \mathrm{~Hz})$, $3.30(1 \mathrm{H}, \mathrm{dd}, J=4.5 \mathrm{~Hz}, 9.5 \mathrm{~Hz}), 7.32(2 \mathrm{H}, \mathrm{d}, J=8.0 \mathrm{~Hz}), 7.81(2 \mathrm{H}, \mathrm{d}, J=8.0 \mathrm{~Hz})$.

${ }^{13} \mathrm{C}$ NMR $\left(\mathrm{CDCl}_{3}\right): \delta-5.7,13.5,18.2,21.6,25.8,32.0,37.7,42.3,65.3,128.1,129.6,135.0$, 144.5.

HRMS Calcd for $\mathrm{C}_{18} \mathrm{H}_{32} \mathrm{NSO}_{3} \mathrm{Si}: 370.1872$. Found: 370.1859 .

\section{Synthesis of 2(S)-[2-(tert-butyl-dimethyl-silanyloxy)-1(S)-methyl-ethyl]-5-methylene-1- (toluene-4-sulfonyl)-piperidine, 11.}

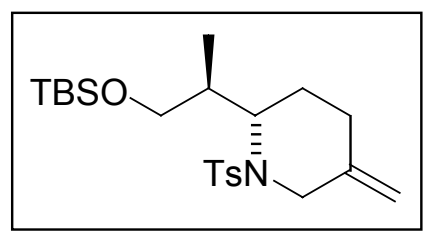

Formation of $0.07 \mathrm{M}$ palladium catalyst batch: A flask was charged with palladium acetate (20 mg, $0.089 \mathrm{mmol}, 1$ eq.), 1,3-bis(diphenylphosphino)propane (92 mg, $0.22 \mathrm{mmol}, 2.5$ eq.) and THF $(1.28 \mathrm{ml})$ to give a yellow solution.

To a solution of aziridine 10 (300 mg, $0.81 \mathrm{mmol}, 1$ eq.) in tetrahydrofuran was added 2[(trimethylsilyl)methyl]-2-propen-1-yl acetate $(227 \mathrm{mg}, 1.2 \mathrm{mmol}, 1.5 \mathrm{eq}$.$) and an aliquot of$ $0.07 \mathrm{M}$ palladium catalyst solution $(1.14 \mathrm{ml}, 0.081 \mathrm{mmol}, 0.1 \mathrm{eq}$.$) and the reaction mixture$ heated at reflux for $16 \mathrm{~h}$. The solvent was removed in vacuo and the residue purified by flash chromatography to give piperidine 11 (141 mg, 41\%) and 12 (117 mg, 30\%).

$[\alpha]_{\mathrm{D}}=+24\left(c=1.4, \mathrm{CH}_{2} \mathrm{Cl}_{2}\right)$.

IR $\left(\mathrm{CH}_{2} \mathrm{Cl}_{2}\right)$ : 3075 (m), 2957 (s), 2857 (s), 1918 (w), 1806 (w), 1734 (s), 1655 (m), 1598 (s), 1472 (s), 1338 (s), 1251 (s), 1160 (s), 1092 (s), 910 (s) cm . $^{-1}$.

${ }^{1} \mathrm{H}$ NMR $\left(\mathrm{CDCl}_{3}\right): \delta 0.06(3 \mathrm{H}, \mathrm{s}), 0.07(3 \mathrm{H}, \mathrm{s}), 0.91(9 \mathrm{H}, \mathrm{s}), 0.95(3 \mathrm{H}, \mathrm{d}, J=6.5 \mathrm{~Hz}), 1.11-1.30$ $(1 \mathrm{H}, \mathrm{m}), 1.56-1.70(1 \mathrm{H}, \mathrm{m}), 1.81-1.93(1 \mathrm{H}, \mathrm{m}), 2.09-2.26(2 \mathrm{H}, \mathrm{m}), 2.41(3 \mathrm{H}, \mathrm{s}), 3.33(1 \mathrm{H}$, dd, $J=8.5,10 \mathrm{~Hz}), 3.58-3.82(3 \mathrm{H}, \mathrm{m}), 4.33(1 \mathrm{H}, \mathrm{d}, J=16 \mathrm{~Hz}), 4.64(1 \mathrm{H}, \mathrm{br}, \mathrm{C}=\mathrm{CHH}), 4.76(1 \mathrm{H}$, br, $\mathrm{C}=\mathrm{CHH}), 7.24(2 \mathrm{H}, \mathrm{d}, J=8.0 \mathrm{~Hz}), 7.69(2 \mathrm{H}, \mathrm{d}, J=8.0 \mathrm{~Hz})$.

${ }^{13} \mathrm{C}$ NMR $\left(\mathrm{CDCl}_{3}\right): \delta-5.4,14.1,18.3,21.5,24.8,26.0,27.3,34.8,47.2,50.0,65.6,110.2$, $127.5,129.4,138.0,141.0,142.8$.

HRMS Calcd for $\mathrm{C}_{22} \mathrm{H}_{38} \mathrm{NSO}_{3} \mathrm{Si}: 424.2341$. Found: 424.2377. 
Acetic acid 4-(tert-butyl-dimethyl-silanyloxy)-3(S)-methyl-2(R)-[(2-methyl-allyl)-(toluene-4sulfonyl)-amino]-butyl ester, 12.

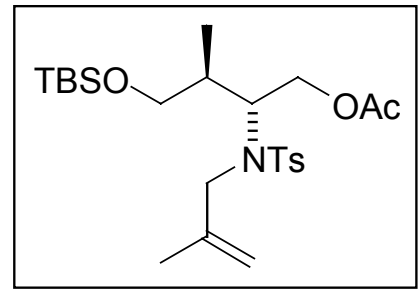

$[\alpha]_{D}=+10\left(c=1.8, \mathrm{CH}_{2} \mathrm{Cl}_{2}\right)$.

IR $\left(\mathrm{CH}_{2} \mathrm{Cl}_{2}\right): 2931$ (s), 2858 (s), 1739 (s), $1599(\mathrm{~m}), 1472$ (s), 1337 (s), 1245 (s), 1093 (s) cm 1.

${ }^{1} \mathrm{H}$ NMR $\left(\mathrm{CDCl}_{3}\right): \delta 0.00(3 \mathrm{H}, \mathrm{s}), 0.01(3 \mathrm{H}, \mathrm{s}), 0.88(9 \mathrm{H}, \mathrm{s}), 0.95(3 \mathrm{H}, \mathrm{d}, J=6.5 \mathrm{~Hz}), 1.67(3 \mathrm{H}$, s), $1.88(3 \mathrm{H}, \mathrm{s}), 1.95-2.07(1 \mathrm{H}, \mathrm{m}), 2.41(3 \mathrm{H}, \mathrm{s}), 3.26(1 \mathrm{H}, \mathrm{dd}, J=7.5,10.0 \mathrm{~Hz}), 3.47(1 \mathrm{H}$, dd, $J=4.0 \mathrm{~Hz}, 10.0 \mathrm{~Hz}), 3.77(2 \mathrm{H}, \mathrm{s}), 3.88(1 \mathrm{H}, \mathrm{dt}, J=4.0,9.0 \mathrm{~Hz}), 4.11(1 \mathrm{H}, \mathrm{dd}, J=9.0,12.0$ $\mathrm{Hz}), 4.26(1 \mathrm{H}, \mathrm{dd}, J=4.0,12.0 \mathrm{~Hz}), 4.88(1 \mathrm{H}, \mathrm{s}), 4.93(1 \mathrm{H}, \mathrm{s}), 7.27(2 \mathrm{H}, \mathrm{d}, J=8.0 \mathrm{~Hz}), 7.71$ $(2 \mathrm{H}, \mathrm{d}, J=8.0 \mathrm{~Hz})$.

${ }^{13} \mathrm{C}$ NMR $\left(\mathrm{CDCl}_{3}\right): \delta-5.5,-14.6,18.3,20.0,20.8,21.4,25.9,36.8,51.7,58.9,63.3,65.7$, $114.8,127.5,129.4,138.3,142.0,143.1,170.7$.

HRMS Calcd for $\mathrm{C}_{24} \mathrm{H}_{42} \mathrm{NSO}_{5} \mathrm{Si}: 484.2553$. Found: 484.2559 .

\section{Synthesis of $N$-\{1-[2-(t-butyl-dimethyl-silanyloxy)-1-methyl-ethyl]-4-hydroxymethyl-} pent-4-enyl\}-4-methyl-benzenesulfonamide, 14.

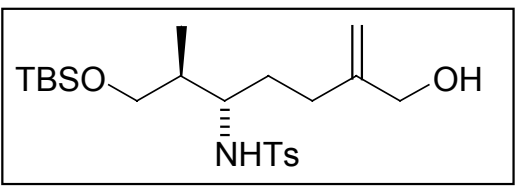

Preparation of the Grignard reagent from 13:

A $100 \mathrm{ml}$ two-necked flask fitted with a reflux condenser and a pressure-equalising dropping funnel was charged with $2.5 \mathrm{M} \mathrm{n}$-butyllithium ( $25 \mathrm{ml}, 61 \mathrm{mmol} 2.6$ eq.). The hexane was removed at reduced pressure with stirring in a warm water bath until a thick yellow oil was obtained. The system was carefully recharged with nitrogen and cooled to $0{ }^{\circ} \mathrm{C}$. Then $\mathrm{Et}_{2} \mathrm{O}$ $(40 \mathrm{ml})$, followed by TMEDA (16 $\mathrm{ml}, 71 \mathrm{mmol}, 3$ eq.) were added via the dropping funnel. 2Methyl-2-propen-1-ol, 13 (2 ml, $24 \mathrm{mmol}, 1$ eq.) was then added dropwise. The reaction was stirred at $0{ }^{\circ} \mathrm{C}$ for $2 \mathrm{~h}$ and then allowed to warm to room temperature overnight. The formation of the dianion as a dark red gummy solid from the orange solution was observed. The solution was cooled to $0{ }^{\circ} \mathrm{C}$ and magnesium bromide $(13 \mathrm{~g}, 70.6 \mathrm{mmol}$, 3 eq.) was added in 3 portions; the precipitation of lithium salts was observed. The resulting suspension was sonicated for 1 hour to help dissolve the remaining organolithium complexes.

Reaction with aziridine 10:

2-[2-(t-Butyl-dimethyl-silanyloxy)-1-methyl-ethyl]-1-(toluene-4-sulfonyl)-aziridine 10 (1.2 g, 3.3 $\mathrm{mmol}, 1 \mathrm{eq})$ in THF $(5 \mathrm{ml})$ was added to the solution of Grignard reagent prepared above at 0 ${ }^{\circ} \mathrm{C}$ and the suspension was sonicated for 30 minutes. After stirring at $\mathrm{rt}$ for a further $48 \mathrm{~h}$, the reaction was quenched with saturated aqueous ammonium chloride then extracted with 
EtOAc. The organic extracts were washed sequentially with saturated aqueous copper sulfate solution and water. The solution was dried over $\mathrm{MgSO}_{4}$ and the solvent removed in vacuo. The residue was purified by flash chromatography (2:1 petroleum ether / ethyl acetate) to give 14 as a colourless oil, $1.15 \mathrm{~g}(80 \%)$.

$[\alpha]_{D}=+20\left(\mathrm{c}=1.0, \mathrm{CH}_{2} \mathrm{Cl}_{2}\right)$.

IR (film): 3457 (br), 3292 (br), 2929 (m), 2857 (m), $1652(\mathrm{w}) \mathrm{cm}^{-1}$.

${ }^{1} \mathrm{H} \mathrm{NMR}\left(\mathrm{CDCl}_{3}\right): \delta 0.0(6 \mathrm{H}, \mathrm{s}), 0.66(3 \mathrm{H}, \mathrm{d}, J=7.0 \mathrm{~Hz}), 0.87(9 \mathrm{H}, \mathrm{s}), 1.52-1.72(4 \mathrm{H}, \mathrm{m}), 1.98$ $(2 \mathrm{H}, \mathrm{t}, J=6.0 \mathrm{~Hz}), 2.37(3 \mathrm{H}, \mathrm{s}), 3.18-3.29(1 \mathrm{H}, \mathrm{m}), 3.31(1 \mathrm{H}, \mathrm{dd}, J=5.5,10.5 \mathrm{~Hz}), 3.61(1 \mathrm{H}$, $\mathrm{dd}, J=3.5,10.5 \mathrm{~Hz}), 3.97(2 \mathrm{H}, \mathrm{s}), 4.75(1 \mathrm{H}, \mathrm{s}), 4.97(1 \mathrm{H}, \mathrm{s}), 5.66(1 \mathrm{H}, \mathrm{d}, J=7.5 \mathrm{~Hz}), 7.23$ $(2 \mathrm{H}, \mathrm{d}, J=8.0 \mathrm{~Hz}), 7.69(2 \mathrm{H}, \mathrm{d}, J=8.0 \mathrm{~Hz})$.

${ }^{13} \mathrm{C} \mathrm{NMR}\left(\mathrm{CDCl}_{3}\right): \delta-5.6,14.0,18.1,21.5,25.8,28.8,31.2,36.9,57.2,65.1,65.9,109.7$, $127.0,129.4,138.6,142.9,148.3$.

HRMS Calcd for $\mathrm{C}_{22} \mathrm{H}_{40} \mathrm{NO}_{4} \mathrm{SS}$ : 442.2447 . Found: 442.2455 .

Synthesis of 2(S)-[2-(t-Butyl-dimethyl-silanyloxy)-1(S)-methyl-ethyl-5-methylene-1(toluene-4-sulfonyl)-piperidine, 11.

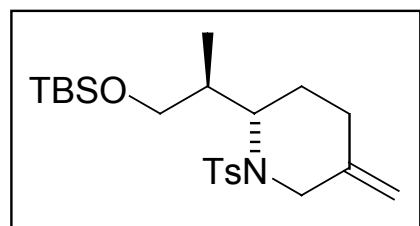

To a solution of $14\left(1.15 \mathrm{~g}, 2.6 \mathrm{mmol}, 1\right.$ eq.) in toluene $(10 \mathrm{ml})$ at $0{ }^{\circ} \mathrm{C}$ was added sequentially tributylphosphine $(0.85 \mathrm{ml}, 3.4 \mathrm{mmol}, 1.3 \mathrm{eq}$.) and ADDP $(0.79 \mathrm{~g}, 3.1 \mathrm{mmol}, 1.2$ eq.). The resulting solution was stirred at room temperature for $16 \mathrm{~h}$ and then concentrated in vacuo. The residue was purified by flash chromatography (12:1 petroleum ether / ethyl acetate) to yield 11 as a colourless oil, $0.95 \mathrm{~g}(87 \%)$. Spectroscopic data was identical to that shown for 11 above.

\section{Synthesis of 2(S)-[5-methylene-1-(toluene-4-sulfonyl)-piperidin-2(S)-yl]-propan-1-ol.}

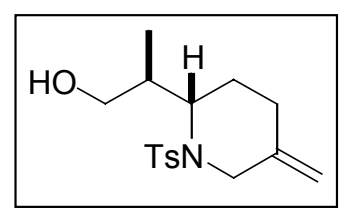

To a solution of piperidine $11(0.95 \mathrm{~g}, 2.3 \mathrm{mmol}, 1$ eq. $)$ in THF (10 ml) was added $1.0 \mathrm{M}$ TBAF $(2.9 \mathrm{ml}, 2.9 \mathrm{mmol}, 1.3 \mathrm{eq}$.). Stirring was continued for $16 \mathrm{~h}$. Water was added to the reaction mixture which was then extracted with EtOAc. Drying with $\mathrm{MgSO}_{4}$ and removal of solvents in vacuo provided the crude oil which was purified by flash chromatography $(3: 1$ petroleum ether / ethyl acetate) to give a colourless oil ( $0.61 \mathrm{~g}, 86 \%)$.

$[\alpha]_{\mathrm{D}}=+68\left(c=0.92, \mathrm{CH}_{2} \mathrm{Cl}_{2}\right)$.

IR $\left(\mathrm{CH}_{2} \mathrm{Cl}_{2}\right): 3527$ (s), 2947 (s), $2883(\mathrm{~m}), 1655(\mathrm{~m}), 1599(\mathrm{~m}), 1462$ (s), 1447 (s), 1336 (s), $1159(\mathrm{~s}), 1090(\mathrm{~s}) \mathrm{cm}^{-1}$. 
${ }^{1} \mathrm{H}$ NMR $\left(\mathrm{CDCl}_{3}\right): \delta 1.04(3 \mathrm{H}, \mathrm{d}, J=7.0 \mathrm{~Hz}), 1.51-1.68(1 \mathrm{H}, \mathrm{m}), 1.92(1 \mathrm{H}, \mathrm{dt}, J=14.0,4.5$ $\mathrm{Hz}), 1.98-2.23(2 \mathrm{H}, \mathrm{m}), 2.42(3 \mathrm{H}, \mathrm{s}), 2.85-2.95(1 \mathrm{H}, \mathrm{m}), 3.42-3.53(1 \mathrm{H}, \mathrm{m}), 3.62-3.75(2 \mathrm{H}, \mathrm{m})$, 3.98-4.06 $(1 \mathrm{H}, \mathrm{m}), 4.40(1 \mathrm{H}, \mathrm{d}, J=16.0 \mathrm{~Hz}), 4.68(1 \mathrm{H}, \mathrm{s}), 4.82(1 \mathrm{H}, \mathrm{s}), 7.26(2 \mathrm{H}, \mathrm{d}, J=8.0$ $\mathrm{Hz}), 7.70(2 \mathrm{H}, \mathrm{d}, J=8.0 \mathrm{~Hz})$.

${ }^{13} \mathrm{C} \mathrm{NMR}\left(\mathrm{CDCl}_{3}\right): \delta 14.4,21.5,24.7,27.1,33.4,47.3,53.9,60.4,63.9,110.7,127.4,129.6$, 140.6, 143.3 .

HRMS Calcd for $\mathrm{C}_{16} \mathrm{H}_{24} \mathrm{NSO}_{3}: 310.1477$. Found: 310.1469 .

\section{Synthesis of $4(R)$-[5-methylene-1-(toluene-4-sulfonyl)-piperidin-2(S)-yl]-pent-2-enoic} acid ethyl ester, 15.

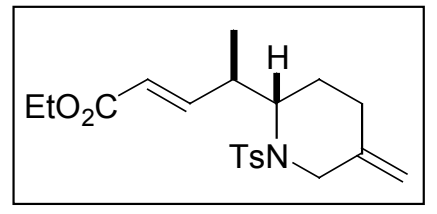

Oxalyl chloride $(0.17 \mathrm{ml}, 2.0 \mathrm{mmol}, 2.25$ eq. $)$ in DCM $(5 \mathrm{ml})$ was stirred at $-78{ }^{\circ} \mathrm{C}$ for $10 \mathrm{~min}$. DMSO $(0.28 \mathrm{ml}, 3.9 \mathrm{mmol}), 4.5$ eq.) was added and stirring continued for a further $30 \mathrm{~min}$. 2(S)-[5-Methylene-1-(toluene-4-sulfonyl)-piperidin-2(S)-yl]-propan-1-ol (270 mg, $0.87 \mathrm{mmol}$, 1.0 eq.) in DCM $(5 \mathrm{ml})$ was added via cannula and stirring continued for $30 \mathrm{~min}$. $\mathrm{Et}_{3} \mathrm{~N}(0.82$ $\mathrm{ml}, 5.9 \mathrm{mmol}, 6.75$ eq.) was added and the mixture allowed to warm to ambient temperature over $1 \mathrm{~h}$. The reaction was quenched with water and extracted with DCM. Drying with $\mathrm{MgSO}_{4}$ and removal of solvents in vacuo provided the crude oil which was used in the next step without any further purification.

Crude aldehyde was dissolved in THF $(10 \mathrm{ml})$ and cooled to $0{ }^{\circ} \mathrm{C} . \mathrm{Ph}_{3} \mathrm{P}=\mathrm{CHCO}_{2} \mathrm{Et}(460 \mathrm{mg}$, $1.3 \mathrm{mmol}, 1.5$ eq.) was added and stirring continued at $\mathrm{rt}$ overnight. Evaporation of the volatiles provided the crude which was purified by flash chromatography (8:1 petroleum ether / ethyl acetate) to give a colourless oil (256 mg, 78\%).

$[\alpha]_{\mathrm{D}}=+20\left(c=1.3, \mathrm{CH}_{2} \mathrm{Cl}_{2}\right)$.

IR $\left(\mathrm{CH}_{2} \mathrm{Cl}_{2}\right): 3063(\mathrm{w}), 2982(\mathrm{~m}), 1713(\mathrm{~s}), 1656(\mathrm{~m}), 1599(\mathrm{~m}), 1446(\mathrm{~m}), 1340(\mathrm{~s}), 1242(\mathrm{~s})$, 1160 (s), $1092(\mathrm{~s}), 1033(\mathrm{~m}), 982(\mathrm{~m}) \mathrm{cm}^{-1}$.

${ }^{1} \mathrm{H}$ NMR $\left(\mathrm{CDCl}_{3}\right): \delta 1.10(3 \mathrm{H}, \mathrm{d}, J=6.5 \mathrm{~Hz}), 1.31(3 \mathrm{H}, \mathrm{t}, J=7.0 \mathrm{~Hz}), 1.35-1.48(1 \mathrm{H}, \mathrm{m}), 1.51-$ $1.79(1 \mathrm{H}, \mathrm{m}), 1.86(1 \mathrm{H}, \mathrm{dt}, J=14.5,5.0 \mathrm{~Hz}), 2.11-2.28(2 \mathrm{H}, \mathrm{m}), 2.41(3 \mathrm{H}, \mathrm{s}), 2.81-2.99(1 \mathrm{H}$, $\mathrm{m}), 3.59(1 \mathrm{H}, \mathrm{d}, J=16.0 \mathrm{~Hz}), 3.78(1 \mathrm{H}, \mathrm{q}, J=4.5 \mathrm{~Hz}), 4.21(2 \mathrm{H}, \mathrm{q}, J=7.0 \mathrm{~Hz}), 4.68(1 \mathrm{H}, \mathrm{br})$, $4.79(1 \mathrm{H}, \mathrm{br}), 5.83(1 \mathrm{H}, \mathrm{d}, J=15.5 \mathrm{~Hz}), 6.92(1 \mathrm{H}, \mathrm{dd}, J=15.5,8.5 \mathrm{~Hz}), 7.24(2 \mathrm{H}, \mathrm{d}, J=8.0$ $\mathrm{Hz}), 7.69(2 \mathrm{H}, \mathrm{d} J=8.0 \mathrm{~Hz})$.

${ }^{13} \mathrm{C} \mathrm{NMR}\left(\mathrm{CDCl}_{3}\right): \delta 14.3,16.5,21.5,24.6,27.1,37.2,47.1,56.8,60.3,110.6,121.3,127.6$, $129.4,138.5,140.6,143.1,150.7,166.3$.

HRMS Calcd for $\mathrm{C}_{20} \mathrm{H}_{28} \mathrm{NSO}_{4}: 378.1739$ Found: 378.1722 . 


\section{Synthesis of 1(R)-methyl-7-methylene-9(aS)-octahydro-quinolizin-4-one, 4.}

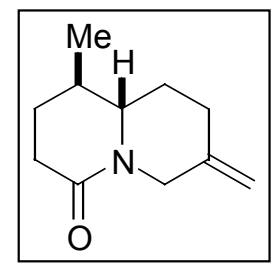

Piperidine 15 (50 mg, $0.13 \mathrm{mmol}, 1$ eq.) in $\mathrm{MeOH}(1.5 \mathrm{ml})$ was added via cannula to a solution of $\mathrm{Mg}$ (194 mg, $4.0 \mathrm{mmol}, 60$ eq.) in $\mathrm{MeOH}(1.5 \mathrm{ml})$ and stirred at $\mathrm{rt}$ for $8 \mathrm{~h}$. The reaction was quenched by addition of $1 \mathrm{M} \mathrm{HCl}$, extracted with EtOAc and dried over $\mathrm{MgSO}_{4}$. The crude product was purified by flash chromatography (2:1 petroleum ether / ethyl acetate) to give a white solid (13 $\mathrm{mg}, 56 \%$ ).

Mp: $73.0-75.0^{\circ} \mathrm{C}$.

$[\alpha]_{\mathrm{D}}=+66\left(c=0.5, \mathrm{CH}_{2} \mathrm{Cl}_{2}\right)$.

IR $\left(\mathrm{CH}_{2} \mathrm{Cl}_{2}\right): 2949(\mathrm{~m}), 2880(\mathrm{w}), 1631$ (s), $1466(\mathrm{~m}), 1420(\mathrm{~m}), 1534(\mathrm{w}), 1307(\mathrm{~m}), 1251$ (m), $1151(\mathrm{w}), 904(\mathrm{~m}) \mathrm{cm}^{-1}$.

${ }^{1} \mathrm{H}$ NMR $\left(\mathrm{CDCl}_{3}\right): \delta 1.08(3 \mathrm{H}, \mathrm{d}, J=6.5 \mathrm{~Hz}), 1.30-1.82(4 \mathrm{H}, \mathrm{m}), 2.06-2.52(5 \mathrm{H}, \mathrm{m}), 2.92(1 \mathrm{H}$, ddd, $J=11.0,8.5,2.5 \mathrm{~Hz}), 3.06(1 \mathrm{H}, \mathrm{d}, J=14.0 \mathrm{~Hz}), 4.77-4.81(1 \mathrm{H}, \mathrm{m}), 4.89-4.93(1 \mathrm{H}, \mathrm{m})$, $5.14(1 \mathrm{H}, \mathrm{dd}, J=14.0,1.5 \mathrm{~Hz})$.

${ }^{13} \mathrm{C} \mathrm{NMR}\left(\mathrm{CDCl}_{3}\right): \delta 19.1,27.9,32.0,32.5,33.2,35.0,48.0,63.0,110.5,141.9,169.1$.

HRMS Calcd for $\mathrm{C}_{11} \mathrm{H}_{18} \mathrm{NO}: 180.1388$. Found: 180.1393.

Synthesis of (1R,7S,9aS)-1,7-dimethyl-octahydro-quinolizin-4-one ${ }^{2}, 16$.

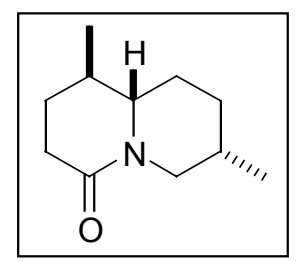

Quinolizinone 4 (12 mg, $0.056 \mathrm{mmol}, 1$ eq.) was dissolved in $\mathrm{MeOH}$ (1 ml) and 10\% Pd/C (12 $\mathrm{mg}, 0.011 \mathrm{mmol}, 0.2$ eq.) added under a $\mathrm{N}_{2}$ atmosphere. The $\mathrm{N}_{2}$ atmosphere was replaced with a $\mathrm{H}_{2}$ atmosphere and the mixture was stirred overnight. Filtration and evaporation of the volatiles provided a white solid as a $6.5: 1$ diastereomeric mixture (12 $\mathrm{mg}, 100 \%)$.

${ }^{1} \mathrm{H}$ NMR $\left(\mathrm{CDCl}_{3}\right): \delta 0.96(3 \mathrm{H}, \mathrm{d}, J=7.0 \mathrm{~Hz}), 1.06(3 \mathrm{H}, \mathrm{d}, J=6.5 \mathrm{~Hz}), 1.40-1.80(7 \mathrm{H}, \mathrm{m}), 1.99$ $(1 \mathrm{H}, \mathrm{m}), 2.34(1 \mathrm{H}, \mathrm{ddd}, J=17.0,11.5,5.5 \mathrm{~Hz}), 2.46(1 \mathrm{H}, \mathrm{ddd}, J=17.0,5.5,4.0 \mathrm{~Hz}), 2.59$ $(1 \mathrm{H}, \mathrm{dd}, J=13.0,3.0 \mathrm{~Hz}), 2.79(1 \mathrm{H}, \mathrm{ddd}, J=11.0,8.5,2.5 \mathrm{~Hz}), 4.55(1 \mathrm{H}, \mathrm{td}, J=13.0,2.0$ $\mathrm{Hz})$.

${ }^{13} \mathrm{C} \mathrm{NMR}\left(\mathrm{CDCl}_{3}\right): \delta 15.9,19.0,27.3,27.4,27.9,30.1,32.0,35.5,47.3,63.6,169.9$. 
Synthesis of (-)-deoxynupharidine, $1^{2}$

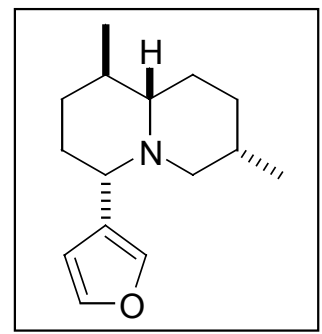

(-)-Deoxynupharidine, 1, was synthesized using the method of Fowler. ${ }^{2}$ The ${ }^{1} \mathrm{H}$ and ${ }^{13} \mathrm{C}$ spectral data were in good agreement with that published: a table of comparative ${ }^{13} \mathrm{C}$ data is shown below for comparison, the ${ }^{1} \mathrm{H}$ and ${ }^{13} \mathrm{C}$ spectra have also been included (see later).

\begin{tabular}{|c|c|}
\hline $\begin{array}{c}\text { Experimental } \\
\text { data }\end{array}$ & $\begin{array}{c}\text { Literature } \\
\text { data }^{3}\end{array}$ \\
\hline 17.5 & 17.6 \\
\hline 19.0 & 19.1 \\
\hline 25.5 & 25.7 \\
\hline 28.4 & 28.7 \\
\hline 30.4 & 30.6 \\
\hline 33.8 & 34.0 \\
\hline 34.9 & 35.0 \\
\hline 35.6 & 35.7 \\
\hline 58.1 & 58.2 \\
\hline 60.0 & 60.3 \\
\hline 69.5 & 69.8 \\
\hline 109.5 & 109.9 \\
\hline 130.0 & 130.4 \\
\hline 139.1 & 139.6 \\
\hline 142.6 & 143.1 \\
\hline
\end{tabular}

\section{References}

1. (a) Gmeiner, P.; Feldman, P. L.; Chu-Meyer, M. Y.; Rapoport, H. J. Org. Chem. 1990, 55, 3068. (b) Baldwin, J. E.; North, M.; Flinn, A. Tetrahedron 1988, 44, 637. (c) Theobald, J. M.; Williams, M. W.; Young, G. T. J. Chem. Soc. 1963, 1927

2. Hwang, Y. C.; Fowler, F. W. J. Org. Chem. 1985, 50, 2719.

3. LaLonde, R. T.; Donvito, T. N.; Tsai, A. I. Can. J. Chem. 1975, 53, 1714. 


$$
\text { 年 }
$$


13C NMR Spectrum of 16

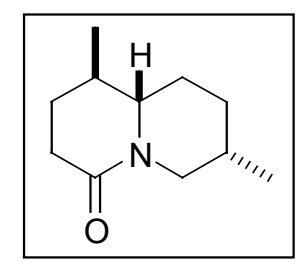

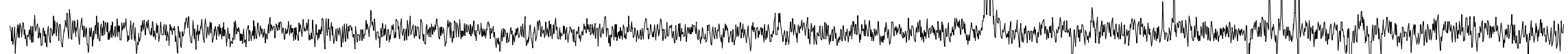




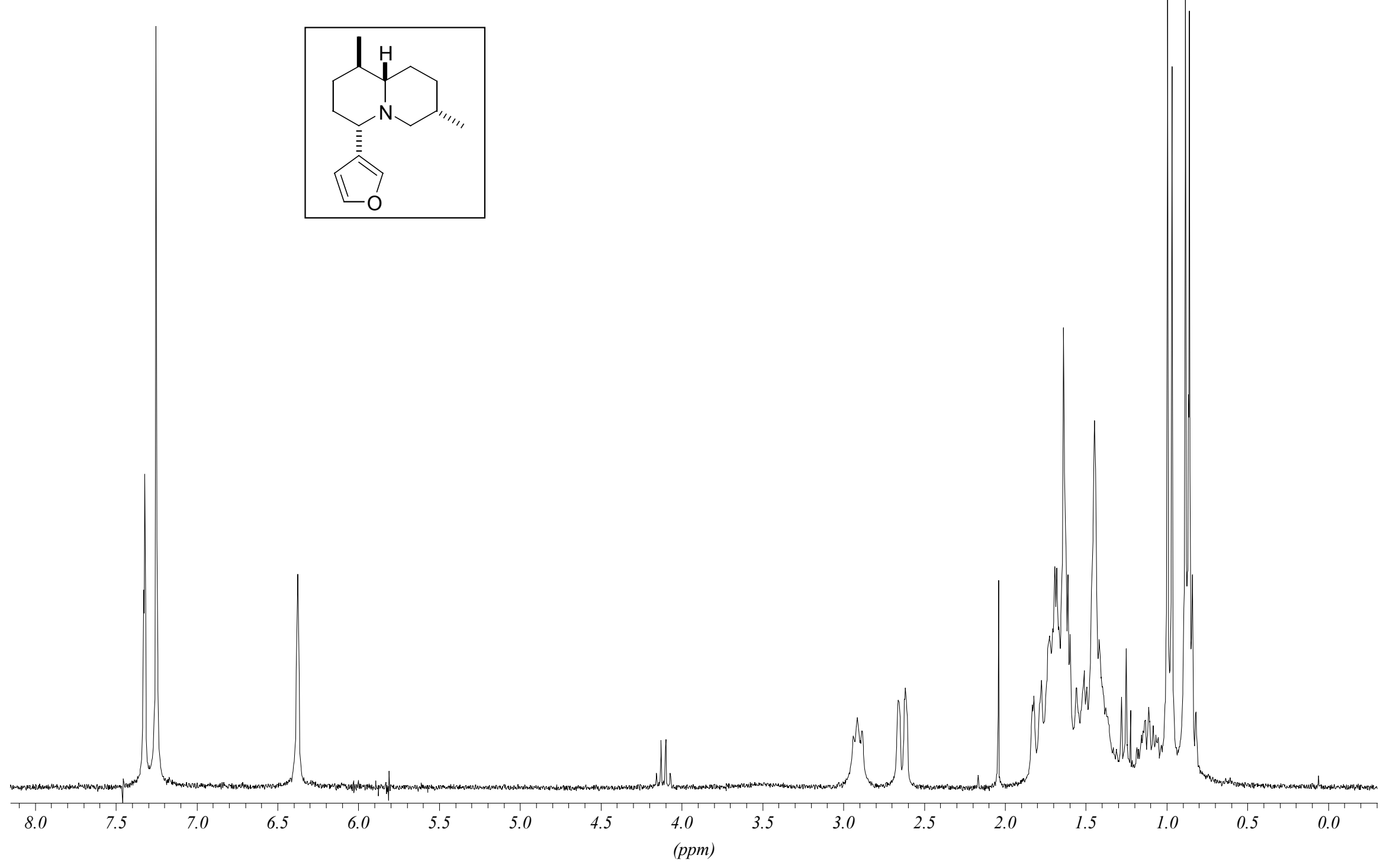




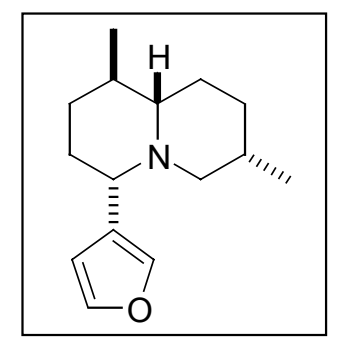

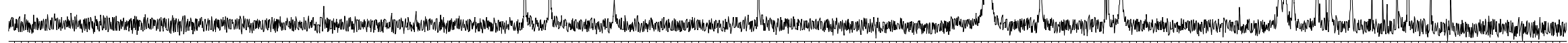

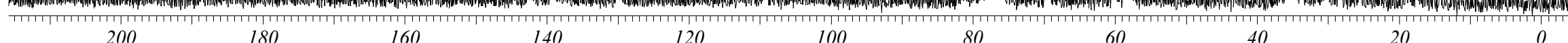

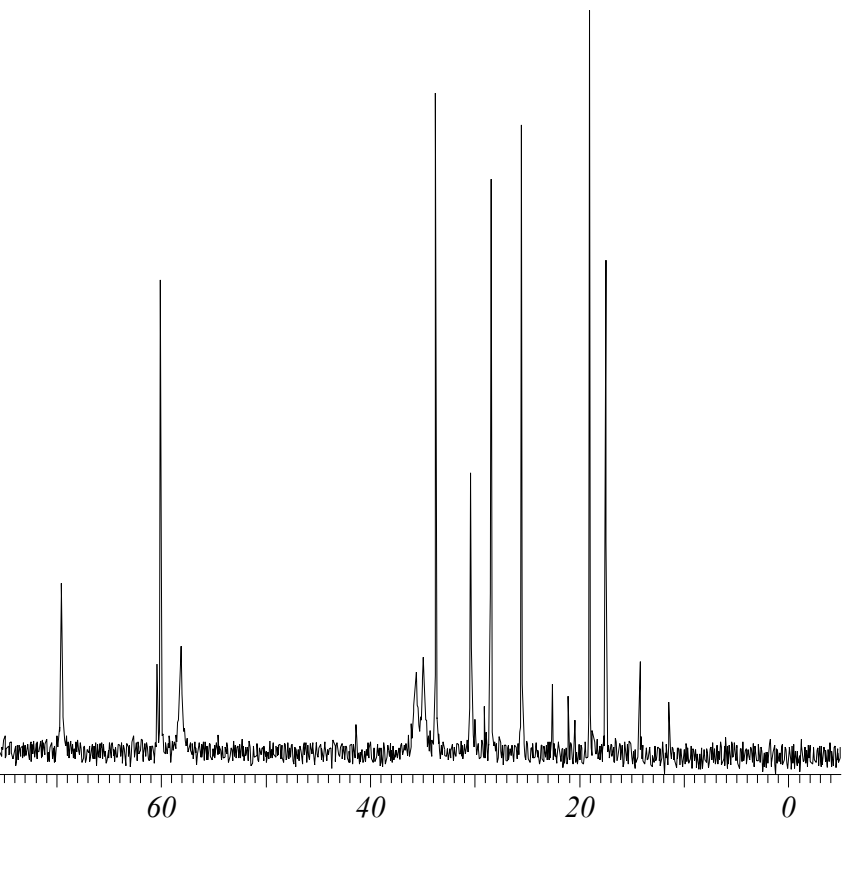

